\title{
Molecular assessment of invasive Streptococcus pneumoniae serotype 1 in Brazil: evidence of clonal replacement
}

Correspondence

Maria-Cristina de Cunto

Brandileone

brandi@ial.sp.gov.br

Received 5 September 2007

Accepted 4 March 2008

\author{
Ana-Carolina Chiou, ${ }^{1}$ Soraya Sgambatti Andrade, ${ }^{2}$ \\ Samanta Cristine G. Almeida, ${ }^{1}$ Rosemeire Cobo Zanella, ${ }^{1}$ \\ Ana-Lúcia Andrade ${ }^{3}$ and Maria-Cristina de Cunto Brandileone ${ }^{1}$ \\ ${ }^{1}$ Bacteriology Department, Adolfo Lutz Institute, São Paulo, Brazil \\ ${ }^{2}$ Division of Infectious Diseases, Federal University of São Paulo, Brazil \\ ${ }^{3}$ Institute of Tropical Pathology and Public Health, Federal University of Goiás, Brazil
}

\section{INTRODUCTION}

The polysaccharide capsule of Streptococcus pneumoniae can be used to define more than 90 pneumococcal serotypes, in addition to acting as the primary virulence determinant for this organism. Some serotypes are mostly associated with nasopharyngeal carriage, whilst others are more likely to be the causative agents of invasive disease (Alannee et al., 2007; Hausdorff et al., 2005).

Serotype 1 ranks among the most prevalent invasive serotypes in many countries (Garcia et al., 2006; Konradsen \& Kaltoft, 2002; McChlery et al., 2005; Porat et al., 2001). In Brazil, since 1977, serotype 1 has been identified as one of the most frequent pneumococci causing severe infections in both adult and paediatric patients (Brandileone et al., 2003). In the period 2000-2005, serotype 1 isolates were ranked fourth (5.6\%) among the principal serotypes identified in Brazilian children up to 6 years of age, preceded by serotypes $14(35.9 \%), 6 \mathrm{~B}(11 \%)$ and $18 \mathrm{C}$ (6.1\%) (Pan-American Health Organization, 2007).

Serotype 1 has specific epidemiological features, including: (i) a low colonization frequency, even in populations in which serotype 1 is a frequent cause of pneumococcal

Abbreviations: $\mathrm{Cl}$, confidence interval; MLST, multilocus sequence typing; OR, odds ratio; SC, Dice similarity coefficient. infections (Laval et al., 2006; Normark et al., 2001; Nunes et al., 2008); (ii) the ability to cause outbreaks in communities and in crowded and closed institutions (Dagan et al., 2000; Leimkugel et al., 2005); (iii) an association with severe episodes of pneumonia and empyema in children (Byington et al., 2006); and (iv) susceptibility to most antimicrobial agents (Klugman \& Koornhof, 1988). Recently, some authors have speculated that isolates of serotype 1, which are known to have a highly invasive potential, behave as primary pathogens, whereas other capsular types demonstrate opportunistic features (Garau \& Calbo, 2007; Hausdorff, 2007).

Molecular typing studies have greatly contributed to epidemiological investigations of pneumococci, mostly focusing on the global spread of penicillin-resistant clones (Brueggemann et al., 2003; Tomasz et al., 1998). Molecular studies of serotype 1, a usually penicillin-susceptible albeit hypervirulent serotype, have been reported less frequently (Brueggemann \& Spratt, 2003; Gonzalez et al., 2004; Normark et al., 2001; Porat et al., 2001). A common factor in these reports was the low genetic diversity among the isolates, which has been associated with the short duration of carriage and/or a low density of this serotype in the nasopharynx, resulting in a reduced opportunity to exchange genes between strains (Brueggemann \& Spratt, 2003; Hausdorff et al., 2005). 
In a study on clonal types of penicillin-susceptible $S$. pneumoniae from Latin American countries, only five serotype 1 strains isolated in 2001 and 2002 from Brazilian individuals were investigated by multilocus sequence typing (MLST), all of which were assigned to sequence type ST304 (Zemlickova et al., 2005). ST304 was recently proposed as clone Sweden ${ }^{1}-40$ by the Pneumococcal Molecular Epidemiology Network (McGee et al., 2001; www.sph.emory.edu/PMEN).

Because of the significant clinical and public health importance of serotype 1 in Brazil (Brandileone et al., 2003), we studied the genetic relatedness of isolates belonging to this capsular type collected from invasive diseases during a three-decade surveillance period in Brazil.

\section{METHODS}

Bacterial isolates. During the period 1977-2005, a total of 8892 pneumococci obtained from normally sterile fluids were recovered through the National Public Health Surveillance Network in Brazil and sent to the Institute Adolfo Lutz, the National Reference Laboratory for meningitis and S. pneumoniae. All isolates were from hospitalized patients. In this collection of pneumococci, a total of 592 $(6.6 \%)$ were identified as serotype 1 by a Neufeld Quellung reaction with antisera obtained from the Statens Seruminstitut (Sorensen, 1993) through the Sistema Regional de Vacunas (SIREVA network), Pan-American Health Organization (Di Fabio et al., 2001). Determination of antimicrobial resistance was carried out as described previously (Brandileone et al., 2006), following the interpretative criteria standardized by the CLSI/NCCLS (2005). All isolates were stored lyophilized in skimmed milk.

In a pilot study conducted to obtain information on an adequate sample size for the current study, we found that the genetic diversity of type 1 pneumococci was slightly higher in the first period (19771984) compared with the last period (2001-2005) of the study. We calculated that a sample size of 134 out of 482 viable serotype 1 strains $(28 \%)$ would be necessary to detect differences in genetic diversity of $10 \%(\alpha=5 \%)$ between the first and last periods of the study. We used convenience sampling (Kleinbaum et al., 1982) to allow the study to include a balanced number of meningitis $(n=62)$ and pneumonia $(n=69)$ isolates and isolates from different geographical regions in Brazil wherever possible. From 1977 to 1984, we studied all viable serotype 1 pneumococci $(n=20)$ as only a small number of isolates were available for this period. For the remaining period, four to ten strains were selected monthly or bimonthly and assembled in three age groups ( $\leqslant 10$ years, $n=79 ; 11-49$ years, $n=38 ; \geqslant 50$ years, $n=17$ ). Pneumococci were recovered from different geographical regions of Brazil: the north-east (three cities), south-east (12 cities) and south (two cities).

Molecular typing. The genotyping of isolates was performed by PFGE as described previously (Brandileone et al., 1998). SmaIrestricted DNA from strain R6 and a Lambda ladder (Biolabs) were used as markers for intra-gel normalization and inter-gel comparison. Representative strains of the 26 international clones (clones 1-26; www.sph.emory.edu/PMEN) and four serotype 1 strains identified previously as sequence type ST304 by MLST (Zemlickova et al., 2005) were also included in the study to investigate their genetic relatedness. Gels were stained with ethidium bromide $\left(5 \mu \mathrm{g} \mathrm{ml}{ }^{-1}\right)$ and photographed in a photo-gel system (Quality One program, version 4.5, Universal HOOD II, Gel Doc EQ; Bio-Rad).
Data analysis. The information related to each isolate was recorded in a data file using EpiInfo software (version 6.04d; Centers for Disease Control and Prevention). The PFGE DNA patterns were initially analysed by visual comparison using the criteria of Tenover et al. (1995) for clustering.

PFGE types (major patterns or lineages designated by capital letters) and subtypes (designated by capital letters with numbers) were defined, respectively, when PFGE profiles differed by more than six fragments and when one to six fragment variations in the same type were observed (Tenover et al., 1995). PFGE patterns were also analysed using BioNumerics software (Applied Maths, version 4.5) by means of the Dice similarity coefficient (SC) with a band-position tolerance of $1.7 \%$ and an optimization of $0.7 \%$. The dendrogram was constructed by UPGMA and a cluster cut-off value of $80 \%$ similarity was ascertained as the same cluster. A $\chi^{2}$ test was applied to compare differences between proportions. The odds ratio (OR) and respective $95 \%$ confidence intervals (95\% CI) were calculated to assess potential factors associated with PFGE types. Variables statistically associated with PFGE types in univariate analysis were adjusted for confounders in a multivariate logistic regression model. $P$ values less than $5 \%$ were considered statistically significant. Data analysis was conducted using SPSS software (version 15.0).

\section{RESULTS AND DISCUSSION}

Among the 134 serotype 1 pneumococci, only two genetic lineages (types A and B) were identified during the period 1977-2005 by visual comparison of the PFGE patterns. Type A comprised 94 isolates (70.2\%) with four subtypes (A1-A4), whereas type B comprised 40 isolates $(29.8 \%)$ with eight subtypes (B1-B8), showing a larger genetic diversity when compared with type A. Subtype A3 was predominant among the type $\mathrm{A}$ isolates and was the most frequent subtype, corresponding to $65 \%$ of the total isolates, followed by subtypes B4 (8.9\%), B2 (8.2\%) and B8 (5.3\%) (Table 1).

Table 1. Distribution of PFGE subtypes of $S$. pneumoniae serotype 1 by geographical region in Brazil from 1977 to 2005

\begin{tabular}{|lcccc|}
\hline \multirow{2}{*}{$\begin{array}{l}\text { PFGE } \\
\text { subtype }\end{array}$} & $\begin{array}{c}\text { No. of isolates } \\
(\%)\end{array}$ & \multicolumn{3}{c|}{ Isolates by region } \\
\cline { 3 - 5 } & & North-east & South-east & South \\
\hline A1 & $4(3.0)$ & - & 4 & - \\
A2 & $2(1.5)$ & - & 2 & - \\
A3 & $87(65.0)$ & 13 & 71 & 3 \\
A4 & $1(0.7)$ & - & 1 & - \\
B1 & $1(0.7)$ & - & 1 & - \\
B2 & $11(8.2)$ & 1 & 10 & - \\
B3 & $4(3.0)$ & - & 3 & 1 \\
B4 & $12(8.9)$ & - & 12 & - \\
B5 & $1(0.7)$ & - & 1 & - \\
B6 & $2(1.5)$ & - & 2 & - \\
B7 & $2(1.5)$ & - & 2 & - \\
B8 & $7(5.3)$ & - & 7 & - \\
Total & $134(100)$ & 14 & 116 & 4 \\
& & & & \\
\hline
\end{tabular}

${ }^{*}$ PFGE subtype according to the criteria of Tenover et al. (1995). 
The genetic similarity of the PFGE types and subtypes established by SC and dendrogram analyses is summarized in Table 2 and shown in Fig. 1. Isolates belonging to subtypes A and B presented SCs higher than $80 \%$, validating types $\mathrm{A}(\mathrm{SC}=85.53 \%)$ and $\mathrm{B}(\mathrm{SC}=84.25 \%)$ as two clonal complexes; the SC for subtype A3 isolates was $97.55 \%$, demonstrating the close relatedness of this group of pneumococci, whilst the SC between subtype A3 and type B isolates was only $72.94 \%$, confirming these two groups of isolates as two distinct genetic lineages.

The stratification of PFGE types by year of isolation is displayed in Fig. 2(a). This analysis allowed us to assess trends in longitudinal clonal distribution. PFGE genotype B was identified from 1977 to 1998 , being predominant in the 1977-1989 period. A similar distribution of B subtypes along that period was observed; subtypes B2 and B4 showed a wide distribution and subtype B2 also occurred as a single isolate in 1995 and 1998. Isolates belonging to type A were identified once in 1980-1981 and then between 1985 and 2005, with a significant predominance of subtype A3 after 1989. Of note, only representatives from genotype A were detected after 1999, $91.5 \%(n=43)$ of which were associated with PFGE subtype A3. Thus these data display a noteworthy temporal switch between two lineages of $S$. pneumoniae serotype 1 in Brazil, and point to the existence of a transitory period that occurred during the years 19841990. After predominating in the first 12 years of the study, genotype B was gradually and effectively replaced by subtype A3, which was established as the major lineage in the last 16 years of the investigation. Analysis of type A lineage $(n=91)$ by clinical diagnosis and age group showed that the increase in type A lineage throughout the study years was mostly detected in isolates from cases of pneumonia (Fig. 2b) and from individuals $<10$ years old (Fig. 2c), when compared with isolates from meningitis and from individuals aged $>10$ years, which remained constant throughout the study period. Indeed, the results of multivariate analysis showed that, in the period 1990 2005 , isolates from pneumonia and isolates from indivi-

Table 2. Genetic relatedness of PFGE types and subtypes of S. pneumoniae serotype 1 isolates from Brazil collected from 1977 to 2005

Results were obtained by computer-assisted analysis, using the UPGMA algorithm based on the Dice SC, with a cluster cut-off value of $80 \%$.

\begin{tabular}{|lc|}
\hline PFGE type and subtype & Genetic relatedness (\%) \\
\hline Subtype A isolates & 85.53 \\
Subtype A3 isolates & 97.55 \\
Subtype A3 and A2 isolates & 96.19 \\
Subtype A3 and A1 isolates & 90.59 \\
Subtype A3 and A4 isolates & 85.87 \\
Subtype B isolates & 84.25 \\
Subtype B and A3 isolates & 72.94 \\
\hline
\end{tabular}

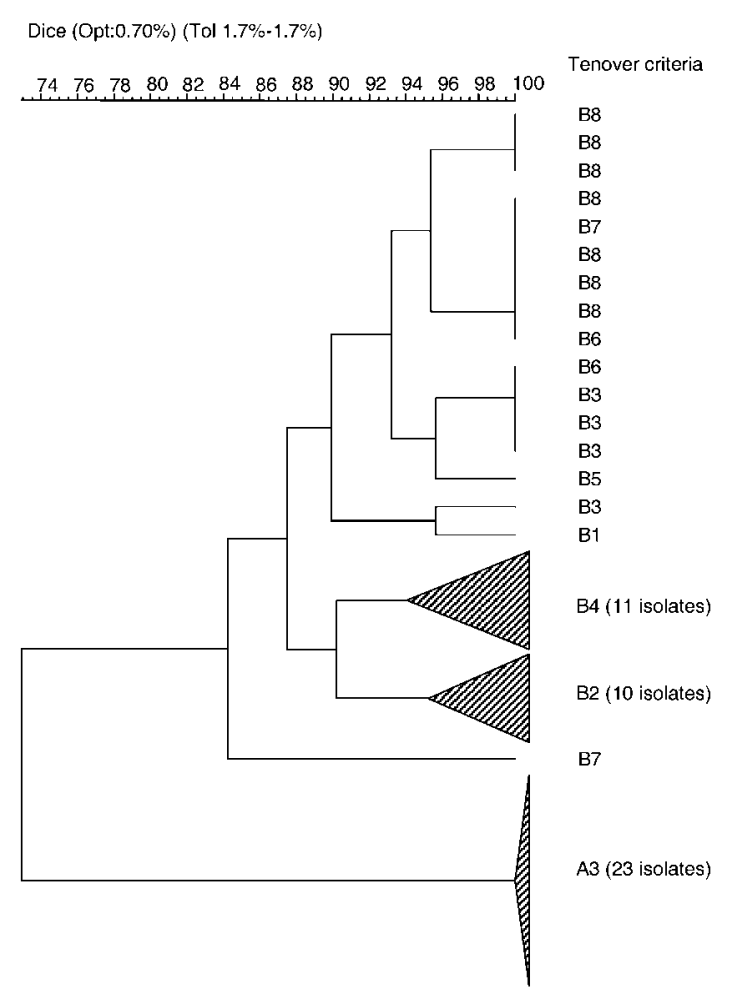

Fig. 1. Dendrogram showing the genetic relatedness of pneumococcal isolates from serotype 1. For the purpose of the dendrogram, S. pneumoniae B subtypes and 23 representatives of the A3 subtype were selected. PFGE classification by visual inspection is given in the right column. Subtypes B2, B4 and A3 are shown as collapsed branches. Isolates with $>80 \%$ similarity were considered to belong to the same clonal group.

duals aged $\leqslant 10$ years were variables independently associated with PFGE type A (Table 3). Therefore, the increase in type A isolates since 1990 in Brazil is strongly associated with isolates recovered from pneumonia cases and from young patients.

The association between serotype $1 \mathrm{~S}$. pneumoniae and pneumonia is well described (Byington et al., 2006). Therefore, it is possible that the specific genotype A of serotype 1 harbours a particular virulence factor for pneumonia. Thus studies on an association between the genetic lineages of $S$. pneumoniae and virulence factors are needed to analyse the molecular epidemiology of pneumococci.

The distribution of PFGE subtypes throughout the geographical regions studied is displayed in Table 1. Subtype A3 first emerged as a single isolate in 1980, collected from a patient with bacterial meningitis in the city of São Paulo in south-east Brazil. Subsequently, this subtype was detected in 17 cities separated by long distances from each other. Although the majority of isolates encountered in this investigation were recovered from the south-eastern region, in particular from São 


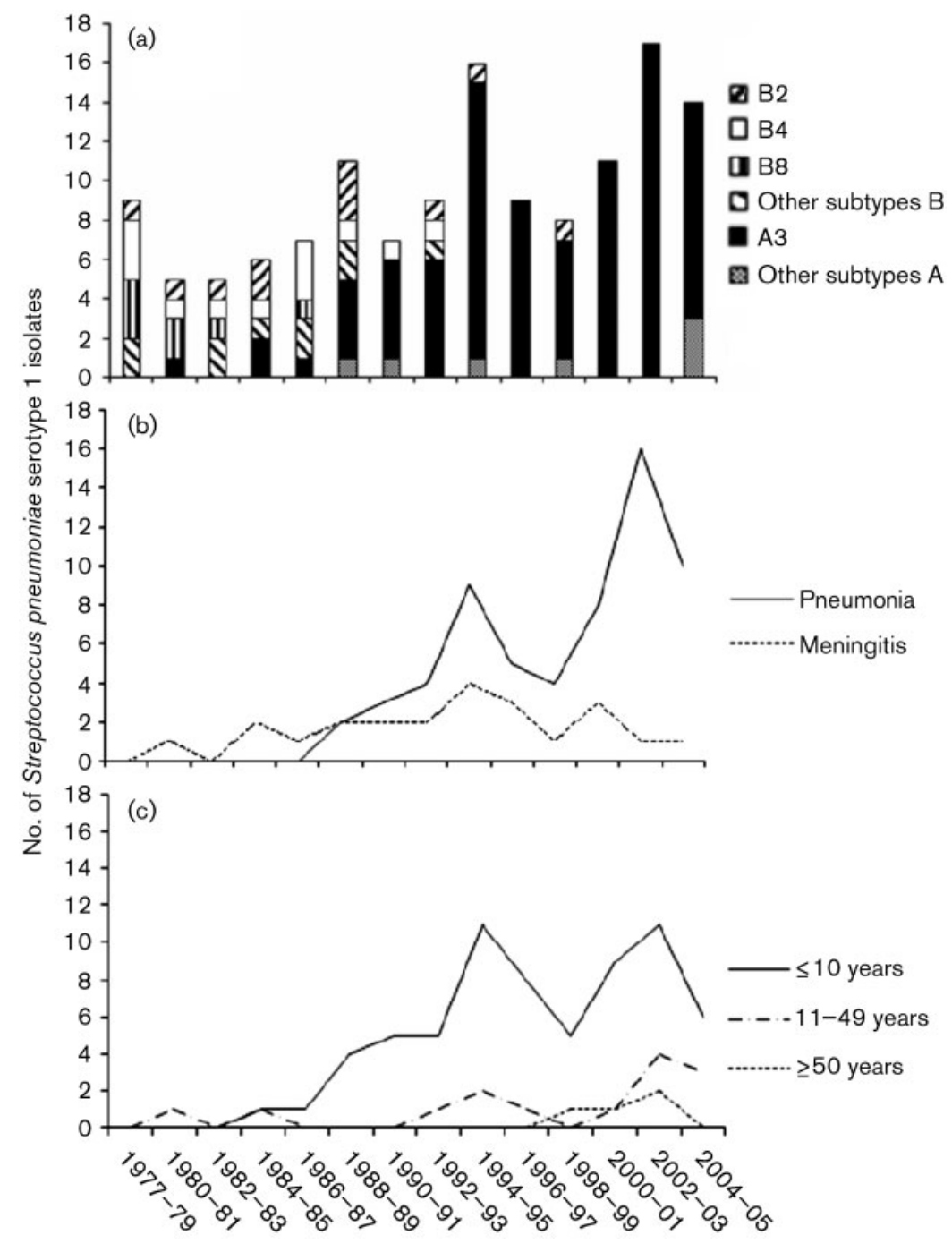

Fig. 2. Distribution of $S$. pneumoniae serotype 1 isolates from 1977 to 2005 in Brazil. (a) PFGE types according to the Tenover criteria; (b) PFGE type A by clinical diagnosis; (c) PFGE type $A$ by age group.

Table 3. Multivariable logistic regression of factors associated with PFGE type of S. pneumoniae serotype 1 from Brazil collected from 1977 to 2005

\begin{tabular}{|c|c|c|c|c|c|}
\hline \multirow[t]{2}{*}{ Variable } & \multicolumn{2}{|c|}{ PFGE type (\%) } & \multirow[t]{2}{*}{ Crude OR $^{\star}(95 \%$ CI $)$} & \multirow[t]{2}{*}{ Adjusted OR (95\% CI) } & \multirow[t]{2}{*}{$P$ value } \\
\hline & A & B & & & \\
\hline \multicolumn{6}{|c|}{ Period of isolation } \\
\hline $1977-1989$ & $9(9.9)$ & $34(85.0)$ & 1 & 1 & \\
\hline 1990-2005 & $82(90.1)$ & $6(15.0)$ & $24.79(6.52-94.26)$ & $25.45(6.81-95.10)$ & 0.000 \\
\hline \multicolumn{6}{|c|}{ Clinical diagnosis } \\
\hline Meningitis & $26(28.6)$ & $36(90.0)$ & 1 & 1 & \\
\hline Pneumonia & $65(71.4)$ & $4(10.0)$ & $4.79(1.22-18.85)$ & $4.70(1.20-18.35)$ & 0.026 \\
\hline \multicolumn{6}{|l|}{ Age group ${ }^{*}$} \\
\hline$>10$ years $\dagger$ & $18(20.2)$ & $27(67.5)$ & 1 & 1 & \\
\hline$\leqslant 10$ years & $71(79.8)$ & $13(32.5)$ & $8.19(3.28-20.74)$ & $6.29(1.72-22.90)$ & 0.005 \\
\hline Total & $91(100.0)$ & $40(100.0)$ & & & \\
\hline
\end{tabular}

*Two samples were missing.

$\dagger$ Adjusted for period of isolation, clinical diagnosis and age group. 
Paulo $(n=90)$, the detection of subtype A3 isolates from three widespread geographical regions in Brazil reflects an extensive dissemination ability of this subtype. This prevalent subtype maintained its uniqueness during the years 2000-2005, without major genetic alterations detectable by PFGE analysis.

The four serotype 1 strains from Brazil previously identified as ST304 by MLST (clone Sweden ${ }^{1}-40$ ) (Zemlickova et al., 2005) were here assigned to subtype A3. Therefore, subtype A3 isolates were identified as belonging to clone Sweden ${ }^{1}-40$ that was present in Brazil about a decade before its widespread dissemination after 1989. The Sweden ${ }^{1}-40$ clone comprises isolates susceptible to penicillin with global dissemination in Latin American, North American and European countries (www.sph. emory.edu/PMEN).

In the period before the temporal switch of the genetic lineages distinguished in this study, no single subtype dominated, as serotype 1 isolates were represented by a large number of B subtypes. Epidemiological factors that may be associated with the replacement of a variety of subtypes B by the clone Sweden ${ }^{1}-40$ are unknown, but this has occurred without antibiotic-pressure selection, as representative isolates of this clone are susceptible to antimicrobial drugs. Clonal expansion of pneumococci in a region is expected to be related to particular characteristics of the community or with a virulence advantage of the pneumococcal lineage (Spratt \& Greenwood, 2000). Examples of clonal expansion associated with serotype 1 isolates have been described recently: the epidemic pneumococcal meningitis that occurred in the Africa meningitis belt, specifically in northern Ghana and Burkina Faso, were caused by serotype 1 isolates of the hypervirulent ST217 clonal complex (Leimkugel et al., 2005; Yaro et al., 2006), and the emergence of a single serotype 1 lineage (ST306) was reported among healthy carriers attending day-care centres in Portugal after the introduction of a seven-valent conjugate vaccine (Nunes et al., 2008).

It is worth noting that, among the serotype 1 isolates from Brazil, three strains were non-susceptible to penicillin. These three particular pneumococci, included in this study and expressing penicillin MICs of between 0.12 and $0.25 \mu \mathrm{g}$ $\mathrm{ml}^{-1}$, all belonged to subtype A3. These pneumococci were isolated in 2004 from pneumonia cases in the city of São Paulo. Thus, considering the fact that serotype 1 is not globally associated with antimicrobial resistance, it is interesting that penicillin resistance appears to have emerged after genetic exchange of penicillin-resistance genes in more recent years among serotype 1 strains belonging to clone Sweden ${ }^{1}-40$. At present, the serotype 1 isolates related to hypervirulent clones are not associated with widespread antimicrobial resistance; however, these clones have great potential to cause invasive disease and may emerge as an antimicrobial-resistant clone. The identification of penicillin-resistant variants of clone
Sweden ${ }^{1}-40$ is of particular concern as it may add a further advantage to such serotype 1 isolates, leading to their emergence in regions where this clone is prevalent as well as in countries where the seven-valent conjugate vaccine is introduced in the vaccination programme (Spratt \& Greenwood, 2000). Serotype 1 is not included in this conjugate vaccine, although more complete formulations of this vaccine (10-valent and 13 -valent vaccines) including this serotype are under clinical trials. The current sevenvalent conjugate vaccine has been licensed in Brazil, but it has not been introduced into the national immunization programme.

In conclusion, this study has reported the genetic relatedness of pneumococci serotype 1 strains isolated over three decades in Brazil, displaying an important replacement of lineage B by the clone Sweden ${ }^{1}-40$, currently responsible for the serotype 1 invasive diseases in this country. This study also confirmed that continuing surveillance is necessary to detect the emergence of pneumococci serotypes and clones so that adequate measures for control of infections caused by pneumococci can be deployed in a timely fashion.

\section{ACKNOWLEDGEMENTS}

We thank the Pan-American Health Organization (PAHO), Washington, DC, USA, for coordinating the SIREVA II study group and for providing the pneumococcal antisera; Marguerite Lovgren from the National Centre for Streptococcus, Edmonton, Canada, for performing the Quality Control Program for SIREVA II; and Lesley McGee from Emory University, Atlanta, USA, for performing the MLST analysis for some Brazilian strains and for a review of the manuscript. This work had financial support from the Instituto Adolfo Lutz (IAL) and Fundação de Amparo a Pesquisa do Estado de São Paulo (FAPESP), São Paulo, Brazil, and from the Canadian International Development Agency (CIDA), Canadá. A.-C. C. was a recipient of a fellowship from FAPESP (grant no. 05/51325-0) and M.-C.C.B. (grant no. 303348/2004-6), R.C.Z. (grant no. 302628/ 2004-5) and A.-L. A. (grant no. 308043/2004-9) received fellowships from the Brazilian National Council for Scientific and Technological Development (CNPq).

\section{REFERENCES}

Alannee, S. R. J., McGee, L., Jackson, D., Chiou, C. C., Feldman, C., Morris, A. J., Ortqvist, A., Rello, J., Luna, C. M. \& other authors (2007). Association of serotypes of Streptococcus pneumoniae with disease severity and outcome in adults: an international study. Clin Infect Dis 45, 46-51.

Brandileone, M. C. C., Di Fabio, J. L., Vieira, V. S. D., Zanella, R. C., Casagrande, S. T., Pignatari, A. C. \& Tomasz, A. (1998). Geographic distribution of penicillin resistance of Streptococcus pneumoniae in Brazil: genetic relatedness. Microb Drug Resist 4, 209-217.

Brandileone, M. C. C., Andrade, A. L. S., Di Fabio, J. L., Guerra, M. L. L. S. \& Austrian, R. (2003). Appropriateness of a pneumococcal conjugate vaccine in Brazil: potential impact of age and clinical diagnosis, with emphasis on meningitis. J Infect Dis 187, 1206-1212.

Brandileone, M. C. C., Casagrande, S. T., Guerra, M. L. L. S., Zanella, R. C., Andrade, A. L. S. S. \& Di Fabio, J. L. (2006). Increase in numbers of $\beta$-lactam-resistant invasive Streptococcus pneumoniae in Brazil and 
the impact of conjugate vaccine coverage. J Med Microbiol 55, 567-574.

Brueggemann, A. B. \& Spratt, B. G. (2003). Geographic distribution and clonal diversity of Streptococcus pneumoniae serotype 1 isolates. J Clin Microbiol 41, 4966-4970.

Brueggemann, A. B., Griffiths, D. T., Meats, E., Peto, T., Crook, D. W. \& Spratt, B. G. (2003). Clonal relationships between invasive and carriage Streptococcus pneumoniae and serotype- and clone-specific differences in invasive disease potential. J Infect Dis 187, 1424-1432.

Byington, C. L., Korgenski, K., Daly, J., Ampofo, K., Pavia, A. \& Mason, E. O. (2006). Impact of the pneumococcal conjugate vaccine on pneumococcal parapneumonic empyema. Pediatr Infect Dis J $\mathbf{2 5}$, 250-254.

CLSI/NCCLS (2005). Performance Standards for Antimicrobial Susceptibility Testing; Fifteenth Informational Supplement. CLSI/ NCCLS document M100-S15. Wayne, PA: Clinical and Laboratory Standards Institute.

Dagan, R., Gradstein, S., Belmaker, I., Porat, N., Siton, Y., Weber, G., Janco, J. \& Yagupsky, P. (2000). An outbreak of Streptococcus pneumoniae serotype 1 in a closed community in southern Israel. Clin Infect Dis 30, 319-321.

Di Fabio, J. L., Castañeda, E., Agudelo, C., De La Hoz, F., Hortal, M., Camou, T., Echániz-Aviles, G., Noemi, M., Barajas, C. \& other authors (2001). Evolution of Streptococcus pneumoniae serotypes and penicillin susceptibility in Latin America, SIREVA-Vigia Group, 1993-1999. PAHO Sireva-Vigía Study Group. Pediatr Infect Dis J 20, 959-967.

Garau, J. \& Calbo, E. (2007). Capsular types and predicting patient outcomes in pneumococcal bacteremia. Clin Infect Dis 45, 52-54.

Garcia, S., Levine, O. S., Cherian, T., Gabastou, J. M., Andrus, J. \& Working Group Members (2006). Pneumococcal disease and vaccination in the Americas: an agenda for accelerated vaccine introduction. Panam J Public Health 19, 340-348.

Gonzalez, B. E., Hulten, K. G., Kaplan, S. L. \& Mason, E. O. (2004). Clonality of Streptococcus pneumoniae serotype 1 isolates from pediatric patients in the United States. J Clin Microbiol 42, 2810-2812.

Hausdorff, W. P. (2007). The roles of pneumococcal serotypes 1 and 5 in paediatric invasive diseases. Vaccine 25, 2406-2412.

Hausdorff, W. P., Feikin, D. R. \& Klugman, K. (2005). Epidemiological differences among pneumococcal serotypes. Lancet Infect Dis $\mathbf{5}$, 83-93.

Kleinbaum, D. G., Kupper, L. L. \& Morgenstern, H. (1982). Design options in observational studies. In Epidemiologic Research: Principles and Quantitative Methods, pp. 51-61. New York: Van Nostrand Reinhold.

Klugman, K. P. \& Koornhof, H. J. (1988). Drug resistance patterns and serogroups or serotypes of pneumococcal isolates from cerebrospinal fluid or blood, 1979-1986. J Infect Dis 158, 956-964.

Konradsen, H. B. \& Kaltoft, M. S. (2002). Invasive pneumococcal infections in Denmark from 1995 to 1999: epidemiology, serotypes, and resistance. Clin Diagn Lab Immunol 9, 358-365.

Laval, C. B., Andrade, A. L. S. S., Pimenta, F. C., Andrade, J. G., Oliveira, R. M., Silva, S. A., de Lima, E. C., Fabio, J. L., Casagrande, S. T. \& Brandileone, M. C. (2006). Serotypes of carriage and invasive isolates of Streptococcus pneumoniae in Brazilian children in the era of pneumococcal vaccines. Clin Microbiol Infect 12, 50-55.

Leimkugel, J., Forgor, A. A., Gagneux, S., Pfluger, V., Flierl, C., Awine, E., Naegeli, M., Dangy, J. P., Smith, T. \& other authors (2005).
An outbreak of serotype 1 Streptococcus pneumoniae meningitis in northern Ghana with features that are characteristic of Neisseria meningitidis epidemics. J Infect Dis 192, 192-199.

McChlery, S. M., Scott, K. J. \& Clarke, S. C. (2005). Clonal analysis of invasive pneumococcal isolates in Scotland and coverage of serotypes by the licensed conjugate polysaccharide pneumococcal vaccine: possible implications for UK vaccine policy. Eur J Clin Microbiol Infect Dis 24, 262-267.

McGee, L., McDougal, L., Zhou, J., Spratt, B. G., Tenover, F. C., George, R., Hakenbeck, R., Heyniewicz, W., Lefèvre, J. C. \& other authors (2001). Nomenclature of major antimicrobial-resistant clones of Streptococcus pneumoniae defined by the pneumococcal molecular epidemiology network. J Clin Microbiol 39, 2565-2571.

Normark, B. M. H., Kalin, M., Ortqvist, A., Akerlund, T., Liljequist, B. O., Hedlund, J., Svenson, S. B., Zhou, J., Spratt, B. G. \& other authors (2001). Dynamics of penicillin-susceptible clones in invasive pneumococcal disease. J Infect Dis 184, 861-869.

Nunes, S., Sá-Leão, R., Pereira, L. C. \& Lencastre, H. (2008). Emergence of a serotype 1 Streptococcus pneumoniae lineage colonising healthy children in Portugal in the seven-valent conjugate vaccination era. Clin Microbiol Infect 14, 82-84.

Pan-American Health Organization (2007). Informe Regional de SIREVA II: Datos por país y por grupos de edad sobre las características de los aislamientos de Streptococcus pneumoniae, Haemophilus influenzae y Neisseria meningitidis, en procesos invasores, 2000-2005. OPS, Documentos Técnicos, Tecnologías Esenciales de Salud, THS/EV, 2007/002. Washington, DC: Pan-American Health Organization (www.paho.org/Spanish/AD/THS/EV/LABS-Sireva.pdf).

Porat, N., Trefler, R. \& Dagan, R. (2001). Persistence of two invasive Streptococcus pneumoniae clones of serotypes 1 and 5 in comparison to that of multiple clones of serotypes $6 \mathrm{~B}$ and $23 \mathrm{~F}$ among children in southern Israel. J Clin Microbiol 39, 1827-1832.

Sorensen, U. B. S. (1993). Typing of pneumococci by using 12 pooled antisera. J Clin Microbiol 31, 2097-2100.

Spratt, B. G. \& Greenwood, B. M. (2000). Prevention of pneumococcal diseases by vaccination: does serotype replacement matter? Lancet 356, 1210-1211.

Tenover, F. C., Arbeit, R. D., Goering, R. V., Mickelsen, P. A., Murray, B. E., Persing, G. H. \& Swaminathan, B. (1995). Interpreting chromosomal DNA restriction patterns produced by pulsed-field gel electrophoresis: criteria for bacteria strain typing. J Clin Microbiol 33, 2233-2239.

Tomasz, A., Corso, A., Severina, E. P., Echaniz-Aviles, G., Brandileone, M. C. C., Camou, T., Castañeda, E., Figueroa, O., Rossi, A. \& Di Fabio, J. L. (1998). Molecular epidemiologic characterization of penicillin-resistant Streptococcus pneumoniae invasive pediatric isolates recovered in six Latin-American countries: an overview. Microb Drug Resist 4, 195-207.

Yaro, S., Lourd, M., Traoré, Y., Njanpop-Lafourcade, B. M., Sawadogo, A., Sangare, L., Hien, A., Quedraogo, M. S., Sanou, O. \& other authors (2006). Epidemiological and molecular characteristics of a highly lethal pneumococcal meningitis epidemic in Burkina Faso. Clin Infect Dis 43, 693-700.

Zemlickova, H., Crisóstomo, M. I., Brandileone, M. C., Camou, T., Castañeda, E., Corso, A., Echaníz-Aviles, G., Pásztor, M. \& Tomasz, A. (2005). Serotypes and clonal types of penicillin-susceptible Streptococcus pneumoniae causing invasive disease in children in five Latin American countries. Microb Drug Resist 11, 195-204. 